\title{
Axillary recurrence after intramammary sentinel lymph nodes metastases with capsular extravasation
}

\author{
Paulo R. De Alcantara Filho', Stephania Martins Bezerra² \\ 'Breast Surgery Department, Hospital Sirio-Libanes, Sao Paulo 01308-050, Brazil. \\ 2Pathology Department, ACCamargo Cancer Center, Sao Paulo 01509-010, Brazil.
}

Correspondence to: Dr. Paulo R. De Alcantara Filho, Breast Surgery Department, Hospital Sirio-Libanes, RUA DONA ADMA JAFET 74, Sao Paulo 01308-050, Brazil. E-mail: institutodemastologia@gmail.com

How to cite this article: De Alcantara Filho PR, Bezerra SM. Axillary recurrence after intramammary sentinel lymph nodes metastases with capsular extravasation. J Cancer Metastasis Treat 2019;5:2. http://dx.doi.org/10.20517/2394-4722.2018.62

Received: 18 Sep 2018 First Decision: 5 Dec 2018 Revised: 14 Dec 2018 Accepted: 25 Dec 2018 Published: 14 Jan 2019

Science Editor: William Schiemann Copy Editor: Cui Yu Production Editor: Huan-Liang Wu

\begin{abstract}
Axillary recurrence is a rare event in patients treated with sentinel lymph node biopsy alone with the majority occurring in the first 5 years after surgery. Intramammary lymph node (IMLN) can be the primary sites of metastasis and sentinel lymph nodes, but the clinical significance, including prognosis and therapeutic approach is yet unclear, even more with capsular extravasation. IMLN metastases are strongly correlated with axillary lymph nodes involvement and therefore a guide for further surgical management of the axillary nodes.
\end{abstract}

Keywords: Breast cancer, sentinel lymph node, lymph node dissection, metastasis, locoregional neoplasm recurrence

\section{INTRODUCTION}

Reported series of patients with intramammary lymph node (IMLN) diagnosed by final histological examination are small in number and clinical significance of metastasis is still unclear ${ }^{[1]}$. We previously reported a case of conservative breast cancer surgery with 3 positive IMLNs demonstrating extracapsular extravasation (ECE). After multidisciplinary discussion, the patient was submitted to mastectomy and no further axillary dissection. Even though the 3 IMLNs were positive, pathology examination did not reveal any signs of malignancy in the mastectomy specimen. The patient underwent adjuvant chemotherapy, radiation and endocrine therapy ${ }^{[2]}$. Here we report the new event after 2 years of follow up.

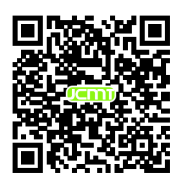



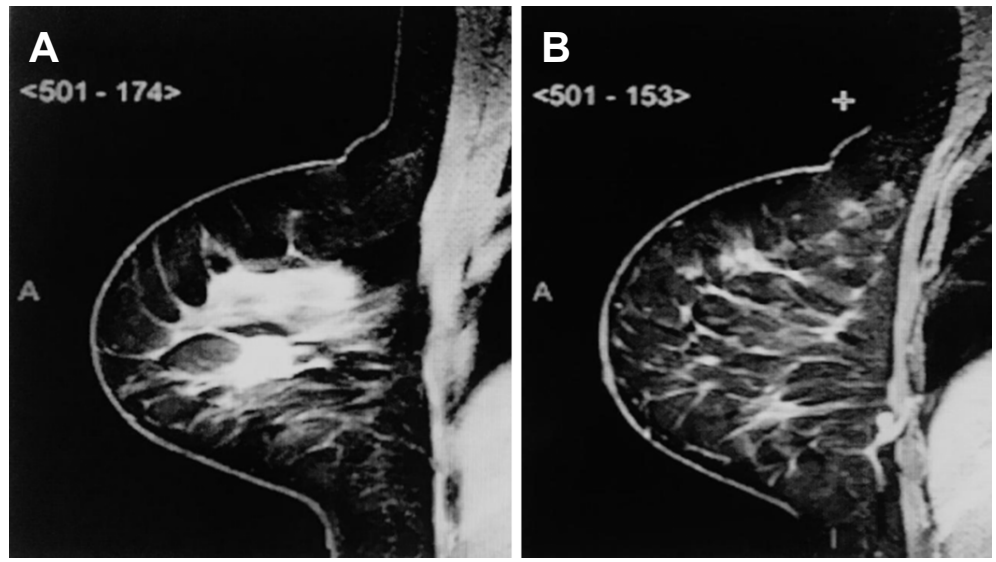

Figure 1. A: Tumor magnetic resonance imaging, axial; B: intramammary sentinel lymph node magnetic resonance imaging, axial view

\section{CASE REPORT}

A 44-year-old, post-menopausal female with a mammogram showing a partially defined lump of the right breast. Breast ultrasound (US) showed a circumscribed hypoechoic lesion, $1.4 \times 1.1 \mathrm{~cm}, 3.8 \mathrm{~cm}$ away from the areolar complex, and also an adjacent hypoechoic microlobulated lesion of $1.4 \times 1.2 \times 1.4 \mathrm{~cm}$, both in the outer quadrants of the right breast. US-guided core-needle biopsy of the lesion revealed a high grade invasive ductal carcinoma (IDC), luminal B (estrogen receptor 30\%, progesterone receptor and human epidermal growth factor receptor type 2 negative, and Ki-67 of $80 \%$ ).

Magnetic resonance imaging (MRI) showed a mass with spiculated contours at early and heterogeneous post-contrast enhancement in the junction of the outer quadrants of the right breast, measuring $1.8 \times 1.6 \times$ $1.5 \mathrm{~cm}$ [Figure $1 \mathrm{~A}$ and B]. In addition, a circumscribed oval mass at early and homogeneous post-contrast enhancement, in lower inner quadrant, measuring $9 \times 5 \times 5 \mathrm{~mm}$ and in close contact with the pectoralis muscle, which seemed to correspond to an IMLN. Second-look US was performed directly to this lymph node, which revealed a suspicious cortical thickening and a decreased hilum. A breast conserving surgery of the index lesion, plus radioguided occult lesion localization of the suspicious lesion in the lower inner quadrant and sentinel lymph node biopsy (SLNB), were performed. The pathology report of the lesions identified an IDC, no special type, histology and nuclear grade III and ductal carcinoma in situ associated. Three axillary sentinel lymph nodes (SLNs) were free of metastases, however the suspected lesion in the lower inner quadrant resulted in 3 IMLNs, all affected by cancer metastases with ECE [Figure 2A and B].

Published literature for IMLNs does not mention capsular leakage, thus, there is no consensus for the best treatment. Pathologists admitted margins in lymph nodes were not frozen, which did not make it feasible to know if the margins of the additional lesions were disease free. Multidisciplinary recommendation was mastectomy without axillary lymph node dissection (ALND) and immediate breast reconstruction with implants, which was performed two weeks after the patient's consent in regards to the unknown probability of further disease in the IMLNs. Pathology report revealed no evidence of malignancy. The patient underwent 4 cycles of anthracycline + cyclophosphamide followed by 12 cycles of taxanes and breast + axillary, internal mammary and supraclavicullar drainage chain radiation therapy + tamoxifen. After 2 years of follow up, the patient suddenly developed axillary pain and fistulization accompanied by fever, and sought the emergency room immediately [Figure 3A]. US and breast MRI showed an axillary lymph node with high T2 signaling, measuring $4.0 \times 3.6 \mathrm{~cm}$ in deep contact with pectoralis muscle and a circumscribed oval mass at early and homogeneous post-contrast enhancement, located in the level 2 of the axilla, measuring $1.0 \times 0.9 \mathrm{~cm}$. 

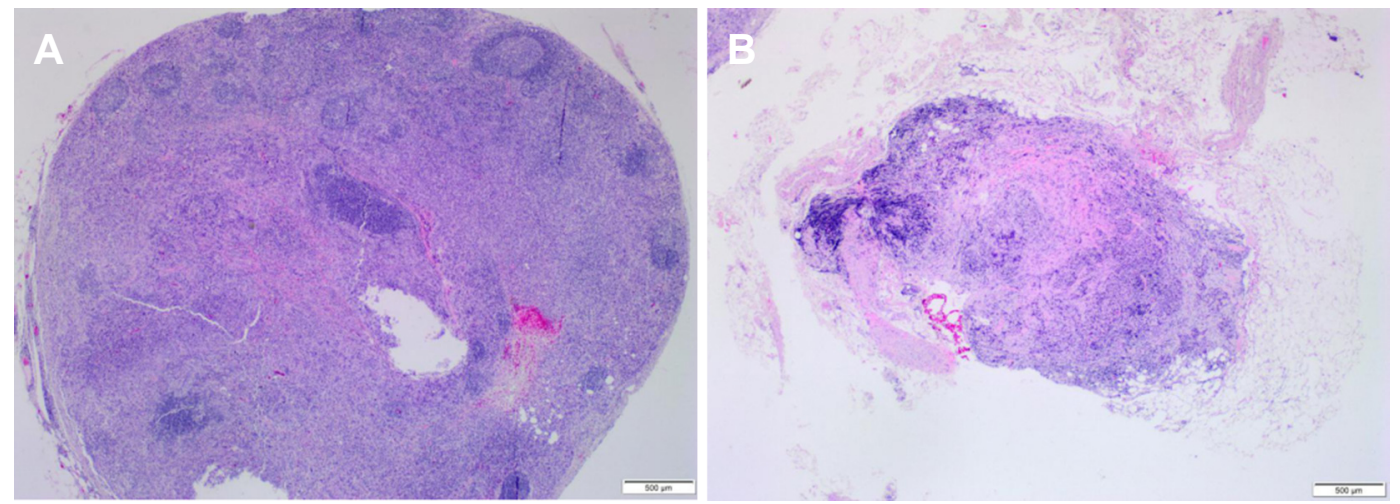

Figure 2. A: Intramammary lymph nodes affected by cancer metastases (3/3): capsular extravasation; B: capsular extravasation
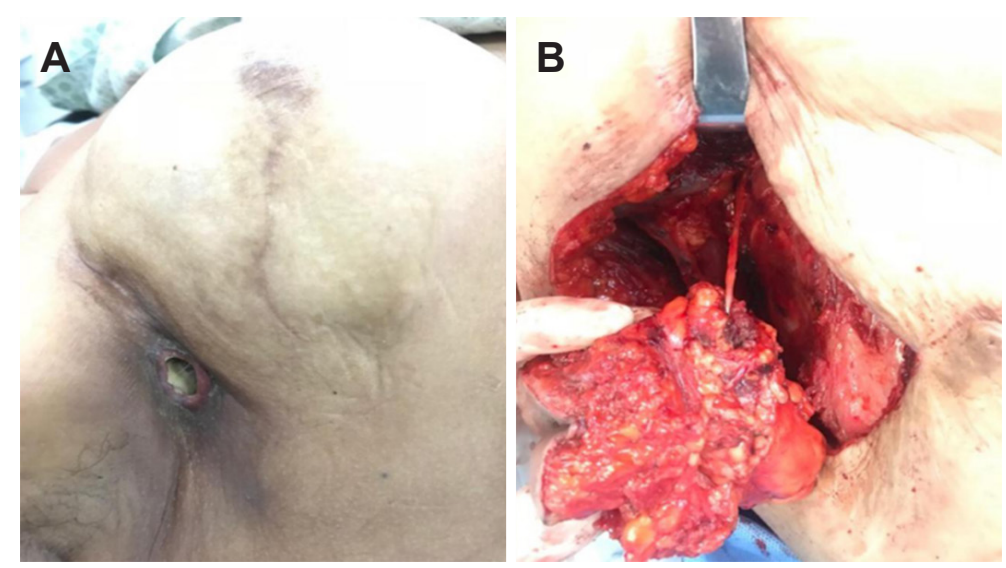

Figure 3. A: Clinical presentation of axillary recurrence with fistulization; B: axillary lymph node dissection

Removal of the implant and ALND with primary closure of the chest wall was performed. Pathology report showed fragment of fibroadipous and muscular tissue compromised by carcinoma, associated to intense chronic inflammatory process abscessed. Skin and 3 axillary lymph nodes level 2 and 3 free of metastasis [Figure 3B].

\section{DISCUSSION}

IMLNs are important because they can be the primary sites of metastasis and SLNs, and when the image is identified, the presence of metastasis is more frequent than in IMLNs detected incidentally ${ }^{[1]}$. They are involved in a variety of clinical situations, including benign lesions, tumor metastases, breast lymphomas and breast cancer, where their importance is not fully established. Nevertheless, there is evidence that IMLNs are an independent factor for poor prognosis, and they may change therapeutic decisions ${ }^{[3]}$. Patients with metastatic IMLNs had more aggressive cancers with lymphatic and vascular invasion as well as increased axillary lymph node metastases ${ }^{[4]}$. In a multicenter study including 33,000 SLN-negative cases from 61 observational series analysis that did not go to ALND reported an axillary recurrence (AR) rate around $0.6 \%{ }^{[5]}$. The NSABP-32 trial showed that SLNB minimized side effects with equivalent survival and regional control than ALND in the SLNB-negative setting and then quickly became the standard of care in early stage breast cancer clinically node-negative ${ }^{[6]}$. AMAROS trial included 1,425 patients with T1-2 breast cancer, no palpable nodes and positive SLNs. Patients were randomized to ALND or axillary radiotherapy and had a median follow up of 6.1 years. No differences in 5-year AR rate was observed between the ALND vs. radiotherapy group ${ }^{[7]}$. 
A retrospective study from the National Cancer Data Base evaluated women with clinically nodenegative breast cancer who had nodal macro or microscopic metastases in the SLN. There were no significant differences in AR with the addition or not of ALND in macrometastasis (1.2 vs. 1, $P=0.4$ ) and micrometastasis $(0.6 \text { vs. } 0.2, P=0.063)^{[8]}$. When IMLN metastases were identified in the breast specimens, it has been suggested that complete ALND may be based on the axillary SLN negative status and thus, ALND can be avoided in this setting ${ }^{[9]}$. Even though our case has not demonstrated malignancy at the surgical site, ECE is considered a sign of worse prognosis, and might be the evidence that leads us to the AR of this patient. Moreover, there is not any standard definition of ECE in the literature. Some authors have used "focal" or "extended" definition with no description regarding adjacent sites of invasion eventually ${ }^{[10]}$. Standard definition of ECE must be identified, because the ECE might be considered by future staging systems ${ }^{[11]}$.

However, it's impossible to conclude that IMLNs metastases featuring ECE might be an independent outcome factor and its clinical meaning is yet controversial and unknown.

\section{DECLARATIONS}

\section{Authors' contributions}

Sample collection: De Alcantara Filho PR, Bezerra SM

Analysis of medical aspects of research, data analysis: De Alcantara Filho PR

\section{Availability of data and materials}

All the data in this article is available to the readers.

\section{Financial support and sponsorship}

None.

\section{Conflicts of interest}

All authors declared that there are no conflicts of interest.

\section{Ethical approval and consent to participate}

Patient consented to participate in a research study.

\section{Consent for publication}

Not applicable.

\section{Copyright}

(c) The Author(s) 2019.

\section{REFERENCES}

1. Pugliese MS, Stempel MM, Cody HS 3rd, Morrow M, Gemignani ML. Surgical management of the axilla: do intramammary nodes matter? Am J Surg 2009;198:532-7.

2. De Alcantara Filho PR, Curi C, Guatelli CS, Osorio CABT, Bezerra SM, et al. Intramammary sentinel lymph node with capsular extravasation in breast cancer. Ann Surg Treat Res 2017;92:376-9.

3. Lee SK, Kim S, Choi MY, Kim J, Lee J, et al. The clinical meaning of intramammary lymph nodes. Oncology 2013;84:1-5.

4. Bolster MJ, Pepels MJ, Wauters CA, Schapers RF, Meijer JW, et al. Is the sentinel lymph node pathology protocol in breast cancer patients associated with the risk of regional recurrence? Eur J Surg Oncol 2013;39:437-41.

5. Houvenaeghel G, Classe JM, Garbay JR, Giard S, Cohen M, et al. Survival impact and predictive factors of axillary recurrence after sentinel biopsy. Eur J Cancer 2016;58:73-82.

6. Mamounas EP. NSABP breast cancer clinical trials: recent results and future directions. Clin Med Res 2003;1:309-26.

7. Donker M, van Tienhoven G, Straver ME, Meijnen P, van de Velde CJ, et al. Radiotherapy or surgery of the axilla after a positive sentinel node in breast cancer (EORTC 10981-22023 AMAROS): a randomised, multicentre, open-label, phase 3 non-inferiority trial. Lancet Oncol 2014;15:1303-10. 
8. Bilimoria KY, Bentrem DJ, Hansen NM, Bethke KP, Rademaker AW, et al. Comparison of sentinel lymph node biopsy alone and completion axillary lymph node dissection for node-positive breast cancer. J Clin Oncol 2009;27:2946-53.

9. Diaz R, Degnim AC, Boughey JC, Nassar A, Jakub JW. A positive intramammary lymph node does not mandate a complete axillary node dissection. Am J Surg 2012;203:151-5.

10. Ilknur GB, Hilmi A, Tülay C, Oguz C, Selma S, et al. The importance of extracapsular extension of axillary lymph node metastases in breast cancer. Tumori 2004;90:107-11.

11. Kanyilmaz G, Findık S, Yavuz BB, Aktan M. The significance of extent of extracapsular extension in patients with T1-2 and N1 breast cancer. Eur J Breast Health 2018;14:218-24. 